\title{
Search for an onset mechanism that operates for both CMEs and substorms
}

\author{
G. L. Siscoe ${ }^{1}$, M. M. Kuznetsova ${ }^{2}$, and J. Raeder ${ }^{3}$ \\ ${ }^{1}$ Center for Space Physics, Boston University, Boston, MA 02215, USA \\ ${ }^{2}$ Space Weather Laboratory, NASA Goddard Space Flight Center, Greenbelt, MD 20771, USA \\ ${ }^{3}$ Space Science Center, University of New Hampshire, Durham, NH 03824, USA
}

Received: 23 October 2008 - Revised: 10 March 2009 - Accepted: 10 March 2009 - Published: 12 August 2009

\begin{abstract}
Substorms and coronal mass ejections have been cited as the most accessible examples of the explosive energy conversion phenomenon that seems to characterize one of the behavior modes of cosmic plasmas. This paper addresses the question of whether these two examples - substorms and CMEs - support or otherwise the idea that explosive energy conversion is the result of a single process operating in different places and under different conditions. As a candidate mechanism that might be common to both substorms and CMEs we use the Forbes catastrophe model for CMEs because before testing it appears to have the potential, suitably modified, to operate also for substorms. The essence of the FCM is a sudden onset of an imbalance of the forces acting on an incipient CME. The imbalance of forces causes the CME to start to rise. Beneath the rising CME conditions develop that favor the onset of magnetic reconnection which then releases the CME and assists its expulsion. Thus the signature of the FCM is a temporally ordered sequence in which there is first the appearance of force imbalance which leads to upward (or outward) motion of the CME which leads to magnetic reconnection under it which expedites rapid expulsion. We look for the FCM signature in the output of two global magnetospheric MHD simulations that produce substorm-like events. We find the ordered sequence of events as stated but with a significant difference: there is no plasmoid prior to the onset of rapid reconnection, that is, there is no counterpart to the incipient CME on which an imbalance of forces acts to initiate the action in the FCM. If this result - that rapid tailward motion precedes the rapid reconnection of substorm expansion - is ultimately verified by other studies, it suggests that a description of the cause of substorm expansion should identify the cause of the preceding rapid tailward motion, since this leads necessarily
\end{abstract}

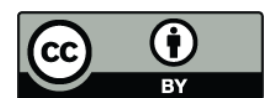

Correspondence to: G. L. Siscoe (siscoe@bu.edu) to rapid reconnection, whatever the reconnection mechanism turns out to be. Clearly then, it is important to identify the cause of the preceding tailward motion.

Keywords. Magnetospheric physics (Storms and substorms) - Solar physics, astrophysics, and astronomy (Flares and mass ejections)

\section{Introduction}

Explosive energy conversion is an apparently universal aspect of the behavior of cosmic plasmas. It is listed with four other such seemingly universal aspects in the report of the US National Research Council titled "Plasma Physics of the Local Cosmos", published in 2004. The other four aspects are discussed under the headings creation and annihilation of magnetic fields, formation of structures and transients, plasma interactions, and energetic particle acceleration. The report notes that there are a number of examples of candidate universal processes in the heliosphere that are accessible to observation, either in situ or by remote sensing. Thus the heliosphere can be used as a local laboratory for studying aspects of the behavior of cosmic plasmas that appear to have universal character. The purpose of this paper is to outline a project focused on substorms and coronal mass ejections (CMEs) to carry out the intent of the NRC report as it relates to explosive energy conversion and to give initial results of the project.

Substorms and CMEs are generally assumed to be manifestations of a process of slow buildup of magnetic energy (e.g., by stretching in a sheared flow field) and sudden conversion of the built up magnetic energy into kinetic forms when the threshold for an instability has been reached. The slow-buildup, sudden-release aspect of the phenomenon is the essence of its universal nature. But does the universal character go beyond just the buildup-release aspect? Is there

Published by Copernicus Publications on behalf of the European Geosciences Union. 
also a common aspect to the instability that converts accumulated magnetic energy suddenly into kinetic forms? Said differently, is the cause of suddenness universal? This is the question that we address here. Do the instabilities that give substorms and CMEs their explosive nature share a common mode of operation or do they operate by means of two distinctly different modes each serving separately and by different, location-specific processes to suddenly release the builtup magnetic energy?

The approach we have taken toward closing in on an answer to the stated question is to survey instabilities (or synonymously, onset mechanisms) that have been proposed to explain the explosive nature of substorms and CMEs and to look among them for mechanisms that can in principle operate both for substorms and CMEs. Such an approach necessarily must overlook onset mechanisms that can operate only for CMEs or only for substorms. However it should be emphasized that just because we must omit for our purpose any mechanism that cannot operate both at the Sun and at the Earth it does not mean that the excluded mechanism might not nonetheless be the actual mechanism that does indeed operate either at the Sun or at the Earth. It only means that a project that sets out to find an onset mechanism common to both substorms and CMEs is obliged to ignore mechanisms that can work for only one.

\section{Criteria for selecting candidate mechanisms}

The stated constraint would seem to eliminate from consideration for our purpose onset mechanisms that depend on a threshold for a specific micro-instability that produces anomalous resistivity to allow to occur rapid unfreezing of the field to the plasma, such as magnetic reconnection, since such thresholds would be specific to local plasma parameters that are considerably different for the two situations (e.g., as discussed in Tajima and Shibata, 1997, Chapter 3, for the Sun; Birn and Hesse, 1992, for the Earth). The universal-inprinciple constraint also removes from consideration onset mechanisms that depend on a mass-loss instability in which a CME goes from negative to positive buoyancy, since this will not work for substorms (e.g., Zhang and Low, 2004). We must ignore as well onset mechanisms that absolutely require triggering, since triggering is not part of current CME scenarios (e.g., Lyons, 1995). (Ignoring triggering for the present purpose is not to deny that triggering of some substorms, perhaps a majority, is an observed fact.) Also out of consideration are onset mechanisms that absolutely require breaking the bonds of an overlying magnetic tether à la the breakout model for CMEs, since such a breakout mechanism is not part of current substorm scenarios (e.g., Antiochos et al., 1999). It will be noticed that our preliminary culling of options excludes from consideration many mechanisms that are actively championed by members of the CME and substorm communities. In fact, it appears that no mechanism in the current menu of proposed onset mechanisms is obviously common to both situations. For the project to proceed, therefore, the search must be expanded in an attempt to identify from available mechanisms one that is in principle potentially able to operate in both situations. We maintain that success here would by itself constitute a useful contribution to the subject of explosive energy conversion. It is a necessary step forward to determine whether, indeed, there is a universal aspect to the instabilities behind the explosive nature of substorms and CMEs.

\section{A test case}

For the stated purpose of identifying from the field of onset mechanisms that have been proposed for either substorms or CMEs, we begin by making a provisional list of features that appear to be common to the two phenomena: 1 . three dynamical stages (buildup, release and propagation), 2. an essential role for the magnetic force (either to restrain or to repel), 3. an essential role for magnetic reconnection, and 4. a characteristic champagne-glass geometry. Some people might wish to add to or subtract from the list as given, but it fairly describes the way substorms are envisioned to occur phenomenologically, and it serves to identify among wellworked-out CME models one that uses only these features and uses no feature that is inherently specific to the Sun, and so, it is a CME model that might also work for substorms. The model is the Forbes catastrophe model (Forbes, 1990; Forbes and Isenberg, 1991). In essence (though not identical with the original version), the model achieves its explosive energy conversion by increasing the magnetic repulsion force on a magnetic bubble (the model's incipient CME) during a "buildup" phase until it overcomes the force holding it to the Sun (gravity or magnetic tension or both). Once the bubble begins to move under magnetic repulsion, the onset stage begins. The champagne-glass geometry promotes rapid magnetic reconnection along the glass stem, and the bubble explodes outward under a positive feedback between outward motion of the bubble and magnetic reconnection driven by the inward motion of plasma to fill the volume vacated by the bubble. The propagation stage then follows.

If, as we are suggesting, the Forbes mechanism has the potential to unify the description of substorm and CME onset mechanisms, then one should be able to identify the mechanism's signature in the numerical output of global MHD simulations of substorms. The mechanism's generic signature is a force imbalance preceding the explosive conversion of magnetic energy into directed kinetic energy. For substorms the force imbalance must be such that the force tending to push the plasma away from Earth (i.e., plasma pressure in this case) exceeds the force tying it to Earth (i.e., magnetic tension). It would, of course, be more convincing to look for a pre-onset force imbalance directly in data instead of in numerical simulations. But to do so would require multi-point 
measurements during a substorm from spacecraft strung out radially along the plasma sheet with possibly more spacecraft than the THEMIS mission contains (as may be inferred from figures shown below), and in any case looking at MHD simulations provides a complementary tack with the advantage of control over the input variables and full 3-D coverage. Moreover, if the force-imbalance signature fails to appear in MHD substorm simulations, the project will have succeeded in eliminating force-imbalance as an onset mechanism common to CMEs and substorms.

\section{A problem of merging $\mathrm{CME}$ and substorm ideas at a mature stage in both fields}

Before proceeding directly to results of MHD simulations of substorms, we should say how the effort recorded here to add something of interest to substorm research relates to the vast and venerable field of work that already exists on substorm modeling. People have been constructing substorm models for nearly forty years; thus it seems highly unlikely to be possible to introduce anything radically new to the field at this time, and the present effort does not do so. Indeed, substorm researchers will recognize in the approach taken here much that is familiar in prior works, especially the classical, semi-empirical model of Hones (1976) and its progression into the near-earth neutral line (NENL) model (e.g., Baker et al., 1996), and the numerical studies of Otto and Schindler (1990), Scholer and Hautz (1991), and Hesse and Birn (1991), to name a few.

But there is a distinguishing and for our purpose important difference between the onset mechanisms of the Forbes CME model and the presently advocated substorm models. The difference is that whereas in substorm models the explosive aspect of substorms is owing to the sudden onset of rapid magnetic reconnection (or some other dissipation process that suddenly unfreezes the field from the plasma) in the Forbes model it is instead the onset of an imbalance between forces holding the incipient CME down and forces pushing it up. The forces involved in the equilibrium that obtains prior to the onset of imbalance are so huge relative to the entrained mass that once the imbalance occurs, there results the greatest accelerations observed in the heliosphere, namely, those of CMEs (e.g., Siscoe et al., 2004). Once the mass is in motion, conditions favorable to magnetic reconnection develop in its wake. Though magnetic reconnection thus follows the onset of acceleration, it is an essential part of the whole process since it allows the CME to escape, and it seems to add significantly to the acceleration by virtue of the reconnection outflow jet.

Therefore in moving from the reconnection-centered focus of present substorm explanations to the Forbes model, one is making a not-very-radical shift in viewpoint, a shift in which forces play a bigger explanatory role than does the mechanism that initiates magnetic reconnection. Reconnection is seen to follow as a necessary consequence of the emergence of a force imbalance that initiates motions that then lead to magnetic reconnection. Thus in the new scenario (to the extent that it is new), force imbalance assumes the initiating role in accounting for the explosive energy conversion of a substorm. The central substorm problem then changes from answering the microphysics question, "What causes the onset of rapid reconnection?" to answering the macrophysics question, "What causes the emergence of force imbalance?" The answer to the macrophysics question would also answer the reconnection question as a by-product.

\section{Results of global MHD simulations}

What then do global MHD substorm simulations say about the relative timing of force imbalance and magnetic reconnection? To obtain a provisional answer this question (provisional in the sense that given the continual improvement in the ability of the codes to represent sub-grid-scale processes, one cannot rule out the possibility that subsequent tests might obtain different results) we use two global MHD simulations performed at the Community Coordinated Modeling Center (CCMC). The outputs of the simulations are available at the CCMC web site. The first simulation used the OpenGGCM code as implemented at CCMC. It is identified at the CCMC web site by the character string UCLA2_UCLA2_060105_3. The run generates a substorm-like condition by flipping in one minute a constant $5 \mathrm{nT}$ IMF from a southward orientation, lasting two hours, to a northward orientation. The simulation is not ideal, however, in that symmetry is not enforced in the cross-tail (y) direction (as it is in the second run discussed below), so that reconnection does not begin on the tail (x) axis. Nonetheless, it rapidly spreads to the tail axis where the pre- and post reconnection conditions are most conveniently displayed. Figure 1 gives a sequence of image taken from the run showing plasma pressure contours and magnetic field lines in the plane containing the dipole axis and the tail axis. The first two frames in the figure reveal a tailward movement of pressure contours that occurs in the eight minutes prior to the onset of reconnection in this plane. By the time of the second frame, one minute before onset, the plasma has already accelerated to $250 \mathrm{~km} / \mathrm{s}$, reflecting the tailward motion of the pressure contours. One minute after onset, in the last frame, the speed has reached $580 \mathrm{~km} / \mathrm{s}$.

To demonstrate directly the emergence of an imbalance between the outward pressure force and the magnetic restraining force that occurs prior to the onset of reconnection, Fig. 2 compares radial profiles along the tail axis of the pressure force $(d P / d x)$ and the magnetic force $(\boldsymbol{J} \times \boldsymbol{B})$ in the $\mathrm{x}$ direction acting on the plasma between 10 and 25 Earth radii down the tail. The red set of profiles corresponds to a forcebalance condition that obtained well before the onset of reconnection and the black set to an out-of-balance condition, 


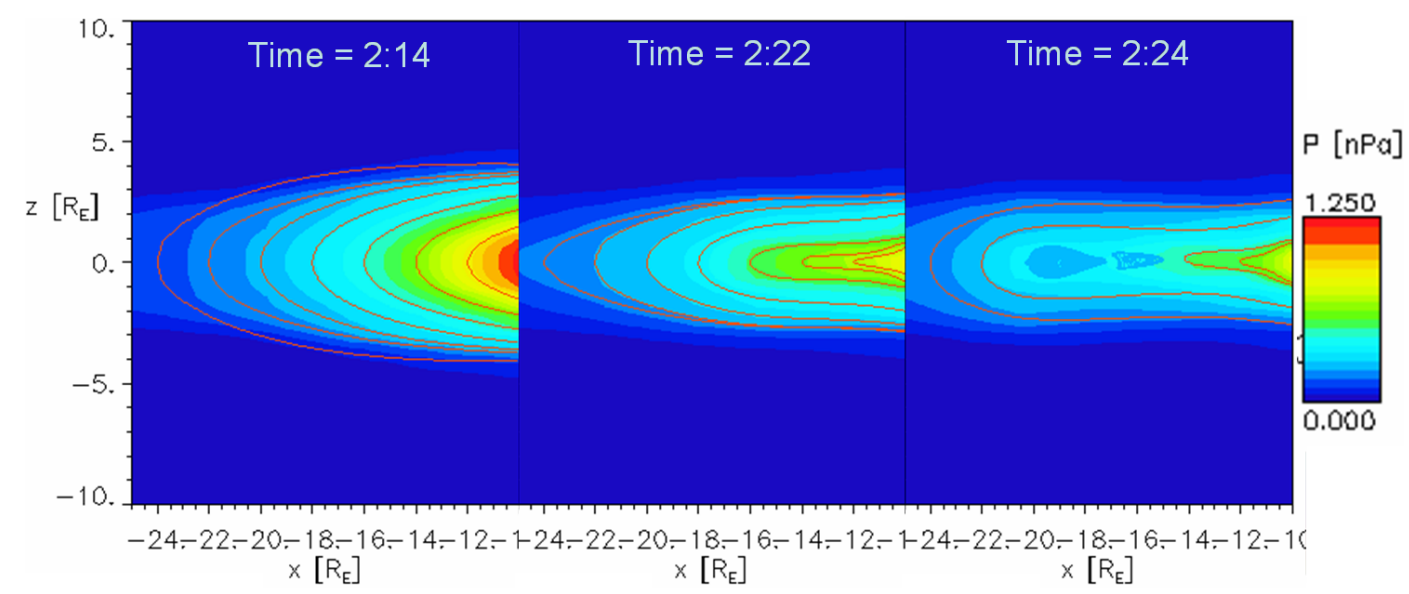

Fig. 1. Three frames from the output of CCMC run UCLA2_UCLA2_060105_3 showing magnetic field lines and contours of plasma pressure in the midnight meridian plane from $10 R_{E}$ to $25 R_{E}$ down the tail. The contours and field lines are changing in time in response to an IMF flip from south to north, which reached the magnetopause at time 2:06. Magnetic reconnection in this plane commenced between 2:22 and 2:24. In the last frame, blue blobs are coiled-up plasmoid field lines. Solar wind conditions for the run are density $=5 \mathrm{n} / \mathrm{cc}, \mathrm{speed}=400 \mathrm{~km} / \mathrm{s}$, temperature $=100000 \mathrm{~K}$, and field strength $=5 \mathrm{nT}$.

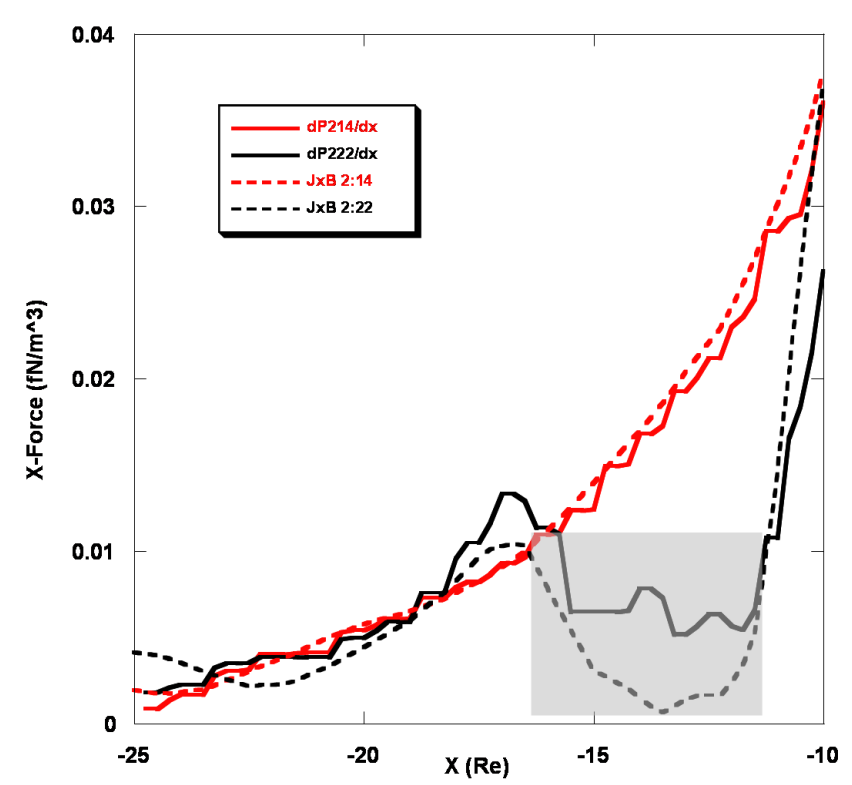

Fig. 2. Corresponding to Fig. 1, this figure shows profiles along the tail axis of the magnetic force $(\boldsymbol{J} \times \boldsymbol{B})$ and the pressure-gradient force (with signed changed for easier comparison) nine minutes prior to reconnection onset (red) and one minute prior to reconnection onset (black). In the gray region the pressure gradient force, which is pushing the plasma away from the Earth, is greater than the magnetic force pulling the plasma toward the Earth.

highlighted in gray, which existed one minute prior to the appearance of reconnection in this plane. In the out-of-balance, pre-onset case, the pressure force and the magnetic force in the highlighted stretch of tail both decrease relative to the in-balance case, but the magnetic force decreases more than the pressure force precisely in the region where magnetic reconnection commences a minute later. It appears, therefore, that we have here a sequence of events that is in important respects consistent with the Forbes mechanism force-imbalance induces motion that leads to magnetic reconnection. What is missing compared to the Forbes model is an incipient plasmoid corresponding to the incipient CME against which forces can be applied and go out of equilibrium (a strict analogy to the Forbes scenario). The sequence of events might be better described as having two phases, a relatively brief preconditioning phase lasting a few minutes during which fast flows arise that stretch the field lines until the second phase occurs initiated by the onset of magnetic reconnection which accelerates the flow to higher speeds. What has been added to the standard substorm scenario, therefore, is the out-of-equilibrium preconditioning phase, which is slower than the reconnection phase but much faster than the classical substorm growth phase. The preconditioning phase might be a substorm analog of the disequilibrium event in the Forbes model.

The description of substorm onset as being a two-phase process - a preconditioning phase with moderately fast tailward flows and a reconnection phase with faster tailward flows - can be applied also to the results obtained with the second simulation to be discussed. In this case the code is the BATS-R-US program as implemented at the CCMC with non-gyrotropic corrections to the Ohm's law to algorithmically simulate sub-grid-scale kinetic dissipation (Kuznetsova et al., 2007). We discuss the results of a run (Masha_Kuznetsova_030106_2) in which a constant 10 nT IMF was held southward resulting in a series of substormlike occurrences in the output of the run. Figure 3 gives a sequence of six images of the plasma sheet in the midnight 


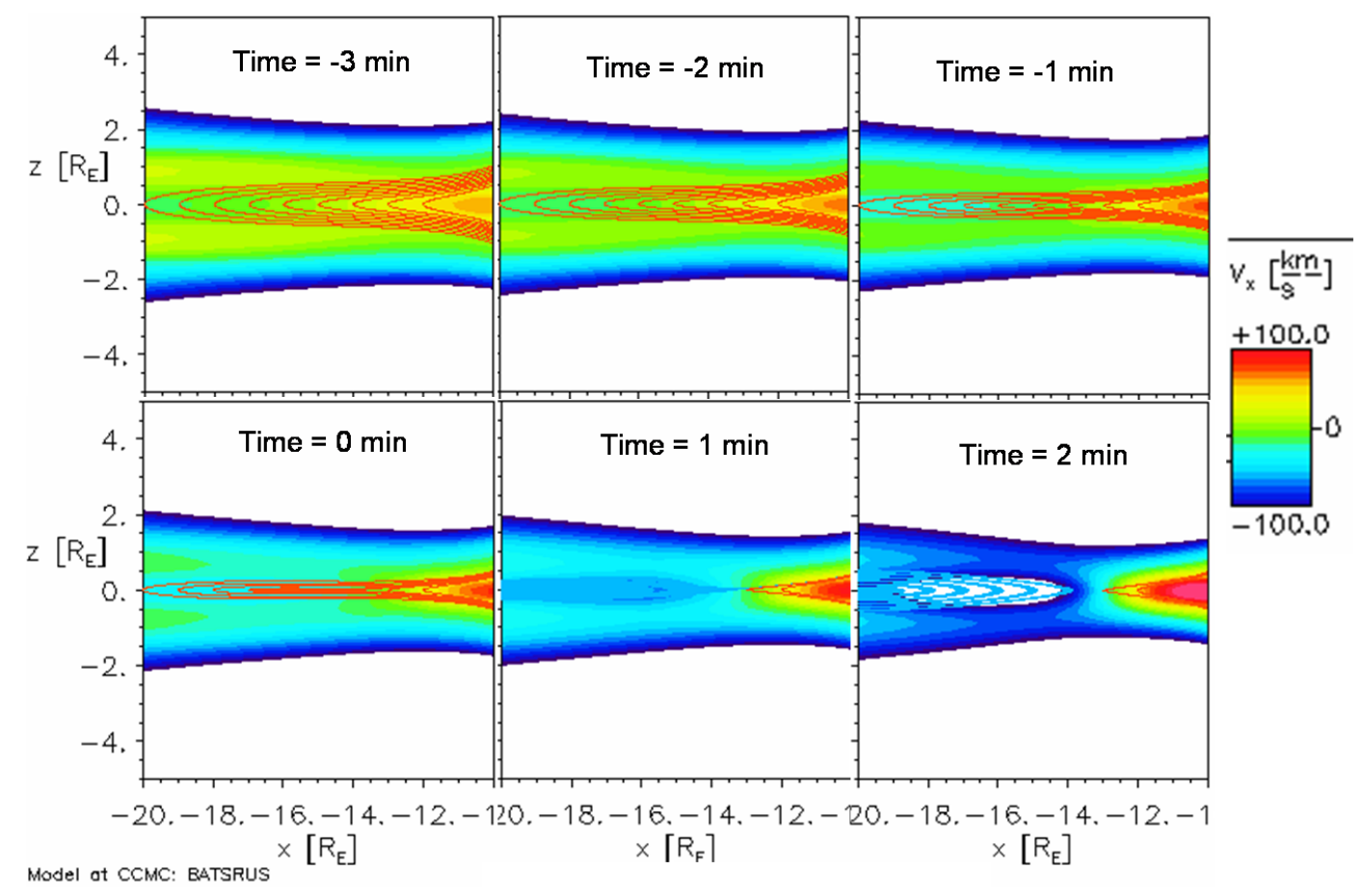

Fig. 3. Six frames from the output of CCMC run Masha_Kuznetsova_030106_2 showing plasma flow speeds in the x-direction and magnetic field lines in the midnight meridian plane from $10 R_{E}$ to $20 R_{E}$ down the tail during a substorm-like event driven by a constant southward IMF. Magnetic reconnection commences at the time of the forth frame. Solar wind conditions for the run are density $=2 \mathrm{n} / \mathrm{cc}$, speed $=$ $400 \mathrm{~km} / \mathrm{s}$, temperature $=200000$, and field strength $=10 \mathrm{nT}$.

meridian plane between 10 and $20 R_{E}$ down the tail during one such substorm-like occurrence. The images show flow contours and magnetic field lines at times separated by one minute starting four minutes prior to the onset of magnetic reconnection. The first two frames reveal no tailward flow in the plasma sheet. Instead there is earthward flow mostly concentrated in the center of the sheet and increasing in speed toward Earth. But the third frame, two minutes prior to the onset of reconnection, shows tailward flow in the center of the plasma sheet beyond about $16 R_{E}$. In the next frame, showing a time when the magnetic field first develops a null point at $15 R_{E}$ but reconnection flows are not yet manifest, the tailward flow envelops the whole plasma sheet beyond about $14 R_{E}$. The last two frames show early stages of the reconnection phase. Peak tailward speed rises from $40 \mathrm{~km} / \mathrm{s}$ in frame 4 to $150 \mathrm{~km} / \mathrm{s}$ in frame 6 . The post reconnection phase with its tailward moving plasmoid of coiled field lines is obviously qualitatively different from the preconditioning phase which seems to be a brief interval of tailward flow causing rapid field-line stretching.

\section{Discussion and conclusions}

We have taken on the project of investigating whether one mechanism rather than two separate mechanisms might oper- ate to cause the aspect of suddenness that characterizes both substorms and CMEs. The first part of the project was to identify candidate mechanisms. We chose the Forbes catastrophe model of CMEs as a promising candidate. The signature of the Forbes mechanism is rapid CME motion preceding magnetic reconnection. The problem was then to identify the mechanism's signature in global MHD simulations of substorms. The attempt was partially successful: rapid motion was indeed found to precede magnetic reconnection, but there was no plasmoid prior to reconnection that would correspond in the Forbes model to the CME that moves prior to reconnection. Instead the whole plasma in the plasma sheet starts to move tailward causing the magnetic field to stretch and ultimately to reconnect rapidly. Reconnection then creates a plasmoid where none existed before and adds considerably to the speed of tailward motion. It might therefore be appropriate to ask whether we should not go in the other direction; is the substorm scenario translatable to CMEs? Perhaps the incipient CME is not necessary, and whatever mechanism it is that causes the fast, pre-reconnection flows in Earth's magnetotail does the same job in a similar geometry in the corona. The problem that this one-versus-two-mechanism project then exposes for the substorm community is to identify the pre-reconnection acceleration mechanism that operates in Earth's magnetotail, that is, to bring this mechanism to a level of understanding similar to that of the Forbes CME 
model so that one can assess its transportability to the corona.

At this point in the discussion we come back to the fact that much modeling of substorms has already been done. So it is possible that the sought-for mechanism has by now been found, but its role as a substorm-counterpart to the Forbes disequilibrium model has gone unrecognized. We are talking here about models that attempt to explain how the current sheet in the plasma sheet in the magnetotail evolves toward a condition that initiates magnetic reconnection. To be responsive to the present need, the model should not be one in which conditions evolve gradually over the duration of the growth phase and, in fact, define the growth phase. Instead it should be one that ends in the manner of an instability that suddenly produces tailward flows and leads quickly to a current sheet so thin that any micro-instability can be tapped to initiate magnetic reconnection. Since the two-phase behavior of substorm-like events that we have identified here in global MHD simulations must be MHD in nature, an MHD type of instability is called for. Among these, the ballooning instability conforms best, perhaps, to the behavior that we have observed, and it has been discussed as a possible mechanism for initiating substorms (Bhattacharjee et al., 1998a, b).

There are other possibilities to the conclusion that we have arrived at here by taking the results of the MHD simulations at face value, namely that a ballooning-like instability precedes magnetic reconnection and this is the substorm counterpart to the Forbes disequilibrium CME model. Instead it could be that a static plasmoid in fact does exist in the tail prior to the explosive phase of a substorm and that the MHD simulations have failed to reproduce it. Then the Forbes disequilibrium mechanism could operate on it as it does on the incipient CME, and the substorm analogy to the CME situation would be more complete than the present analysis suggests. Or as already noted, it could be that a ballooning-like instability operating in a stretched field geometry leading to plasmoid formation and its ejection also applies to CMEs. These are issues that future research will help resolve. Meanwhile, the one-versus-two-mechanisms project pursued here has put in play or reactivated a two-phase substorm-onset scenario ballooning-like instability leading to magnetic reconnection, plasmoid formation and ejection. Should this turn out to be right, future descriptions of substorm onset would emphasize the macroscale (ballooning-like) instability as the initiating event and magnetic reconnection as an inevitable consequence regardless of the specifics of the reconnection microphysics.

Acknowledgements. The work at Boston University was supported in part by the National Science Foundation under grant ATM0220396. Simulation results have been provided by the Community Coordinated Modeling Center at Goddard Space Flight Center through their public Runs on Request system (http://ccmc.gsfc.nasa. gov). The CCMC is a multi-agency partnership between NASA, AFMC, AFOSR, AFRL, AFWA, NOAA, NSF and ONR.

Topical Editor R. Nakamura thanks V. M. Vasyliunas for his help in evaluating this paper.

\section{References}

Antiochos, S. K., DeVore, C. R., and Klimchuk, J. A.: A model for solar coronal mass ejections, Astrophys. J., 510, 485-493, 1999.

Baker, D. N., Pulkkinen, T. I., Angelopoulos, V., Baumjohann, W., McPherron, R. L.: Neutral line model of substorms: Past results and present view, J. Geophys. Res., 101, 12975-13010, 1996.

Bhattacharjee, A., Ma, Z. W., and Wang, X.: Dynamics of thin current sheets and their disruption by ballooning instabilities: A mechanism for magnetospheric substorms, Phys. Plasmas, 5, 2001-2009, 1998a.

Bhattacharjee, A., Ma, Z. W., and Wang, X.: Ballooning instability of a thin current sheet in the high-Lundquist-number magnetotail, Geophys. Res. Lett, 25, 861-864, 1998b.

Birn, J. and Hesse, M.: Magnetotail instability, in: Substorms 1: Proceedings of the First International Conference on Substorms, ESA SP-335, pp. 225-230, 1992.

Birn, J. and Schindler, K: Thin current sheets in the magnetotail and the loss of equilibrium, J. Geophys. Res. 107, Issue A7, pp. SMP 18-1, 1117, doi:10.1029/2001JA000291, 2002.

Birn, J., Schindler, K., and Hesse, M.: Formation of thin current sheets in the magnetotail: Effects of propagating boundary deformations, J. Geophys. Res., 108(A9), pp. SMP 2-1, 1337, doi:10.1029/2002JA009641, 2003.

Forbes, T. G.: Numerical simulation of a catastrophe model for coronal mass ejections, J. Geophys. Res., 95, 11919-11931, 1990.

Forbes, T. G. and Isenberg, P. A.: A catastrophe mechanism for coronal mass ejections, Astrophys. J., 373, 294-307, 1991.

Hesse, M. and Birn, J.: Plasmoid evolution in an extended magnetotail, J. geophys. Res., 96, 5683-5696, 1991.

Hones Jr., E. W.: The magnetotail: It's generation and dissipation, in: Physics of Solar Planetary Environments, edited by: Williams, J., p. 558, American Geophysical Union, 1976.

Kuznetsova, M. M., Hesse, M., Rastätter, L., Taktakishvili, A., Toth, G., De Zeeuw, D. L., Ridley, A., and Gombosi, T. I.: Multiscale modeling of magnetospheric reconnection, J. Geophys. Res., 112(A10), A10210, doi:10.1029/2007JA012316, 2007.

Lyons, L. R.: A new theory for magnetospheric substorms, J. Geophys. Res., 100, 19069-19082, 1995.

Otto, A. and Schindler, K.: Quantitative study of the nonlinear formation and acceleration of plasmoids in the Earth's magnetotail, J. Geophys. Res., 95, 15023-15037, 1990.

Scholer, M. and Hautz, R.: On acceleration of plasmoids in magnetohydrodynamic simulations of magnetotail reconnection, J. Geophys. Res., 96, 3581-34588, 1991.

Siscoe, G., Crooker, N. U., and Elliott, H. A.: Initial-Condition Influences on CME Expansion and Propagation, Solar Phys., 239, 293-316, 2006.

Tajima, T. and Hibata, K.: Plasma Astrophysics, Addison-Wesley, 1997.

Zhang, M. and Low, B. C.: Magnetic energy storage in the two hydromagnetic types of solar prominences, Astrophys. J., 600, 1043-1051, 2004. 